\title{
HIGH-SPEED ROTOR ANALYTICAL DYNAMICS ON FLEXIBLE FOUNDATION SUBJECTED TO INTERNAL AND EXTERNAL EXCITATION
}

\author{
VENELIN S. JiVKOV ${ }^{1}$, EVTIM V. ZAHARIEV ${ }^{2 *}$ \\ ${ }^{1}$ Technical University of Sofia, \\ 8 St. Kliment Ohridski Blvd., 1000 Sofia, Bulgaria \\ ${ }^{2}$ Institute of Mechanics, Bulgarian Academy of Sciences, \\ Acad. G. Bonchev St., Bl. 4, 1113 Sofia, Bulgaria
}

[Received 10 October 2016; Accepted 12 December 2016]

\begin{abstract}
The paper presents a geometrical approach to dynamics simulation of a rigid and flexible system, compiled of high speed rotating machine with eccentricity and considerable inertia and mass. The machine is mounted on a vertical flexible pillar with considerable height. The stiffness and damping of the column, as well as, of the rotor bearings and the shaft are taken into account. Non-stationary vibrations and transitional processes are analyzed. The major frequency and modal mode of the flexible column are used for analytical reduction of its mass, stiffness and damping properties. The rotor and the foundation are modelled as rigid bodies, while the flexibility of the bearings is estimated by experiments and the requirements of the manufacturer. The transition effects as a result of limited power are analyzed by asymptotic methods of averaging. Analytical expressions for the amplitudes and unstable vibrations throughout resonance are derived by quasi-static approach increasing and decreasing of the exciting frequency. Analytical functions give the possibility to analyze the influence of the design parameter of many structure applications as wind power generators, gas turbines, turbo-generators, and etc. A numerical procedure is applied to verify the effectiveness and precision of the simulation process.

Nonlinear and transitional effects are analyzed and compared to the analytical results. External excitations, as wave propagation and earthquakes, are discussed. Finite elements in relative and absolute coordinates are applied to model the flexible column and the high speed rotating machine. Generalized Newton - Euler dynamics equations are used to derive the precise dynamics equations. Examples of simulation of the system vibrations and nonstationary behaviour are presented.
\end{abstract}

KEY WORDS: Multibody systems, elastic bodies, analytical dynamics, rotor dynamics, transition effects, limited power. 


\section{INTRODUCTION}

Rotating machines are basic units in many structures and industrial applications. Most of them are on-board machines that cause inertial excited vibrations and nonstationary phenomena. The automobile engines and kinetic storage devices, aircraft turbines, the large engines and turbines of ships and etc., are only few examples of rotors on moving platforms. The main aim of the rotor balancing is to minimize the centrifugal forces and the inertia excited vibrations, but this approach cannot solve the problem generally. Rotor behaviour could be also influenced by the base movement, that leads to undesirable vibrations and is one of the main reasons for non-stationary phenomena.

In [1], the dynamic behaviour of an asymmetric rotor subjected to translational base excitation is analyzed. An original spectral approach to approximate the rotor response is developed. Lee et al. [2] proposed a Finite Element (FE) model of a rotor considering shock base excitations. They applied the state-space Newmark method and average velocity concept. The authors presented also experimental investigation and compared it with the results.

Some other works incorporated the foundation flexibility into the rotor dynamics. Kang et al. [3] studied the effect of the flexible support (foundation) on the dynamic characteristics of the rotor-bearing systems, using FE modelling.

Recently, a wide variety of phenomena regarding industrial rotor dynamic systems, whose mountings are subjected to movement have been studied [4]. In [5], a general theory of dynamic systems analysis under kinematics excitation is proposed. The results of unsteady dynamic analysis of one degree of freedom system and a rotor supported by non-linear journal bearings are presented. In [6], a detailed survey of the scientific literature regarding the subject was presented.

A brief glance at the papers, discussing the rotor dynamics reveals the strong tendency of application of numerical methods and FE theory to dynamic simulation of rigid and flexible systems with rotors installed. Unfortunately, the geometrical methods that give exact or approximate analytical expressions of the dynamic equations are very often neglected. Recently, this problem was stated among the scientific community and the efforts were directed towards attracting the researchers to development of geometrical and analytical methods for multibody systems dynamics simulation. Some recent papers regarding geometrical approach and principle of the kinetic energy conservation are published by Mukherjee et al. [7, 8]. They analyzed the vibration of elastic rotating shaft with in-span discrete external damping. The analysis is being carried out through extended Lagrangian formulation for continuous system, considered as one-dimensional. They proposed formulation of umbra-Lagrangian density and external damping using extended Noether's theorem 
[9]. This theorem, proved in 1915 by Emmy Noether, was praised by Einstein as a piece of "penetrating mathematical thinking". Umbra- Lagrangian formulation is successfully applied for flexible systems, taking into account the internal and external damping, as well as, the stiffness of the flexible bodies.

Actually, the numerical methods for dynamics simulation of rigid and flexible system propose many advantages and possibilities for analysis of complex systems with many degrees of freedom and nonlinear effects, and are successfully applied in design process. But the outcomes of the computations are only to visualize the results and to convince the engineers that they are in the right direction, but not to poof in hundred percents their conclusions. Although some restrictions of their application, the geometrical methods could lead to fundamental conclusions and proofs. The analytical functions derived could clearly show the influence of the design parameters on the system behaviour. The numerical methods, on the contrary to the analytical, in many cases cannot display the continuous flow of processes and phenomena and could only display the system functionality for particular parameters. Of course, varying the parameters conclusions could be performed, but they are not general. One of the advantages of the numerical methods is the possibility for simulation of nonlinear phenomena and this is the case in the paper taking into account external excitations, caused by an earthquake.

The present paper deals with the inertia excited vibrations, due to high sped rotor with considerable inertia properties and mass. The rotor is mounted on a high vertical pillar. Flexibility of the structure and the basement on which the rotating machine is installed has an impact onto the transitional and non-stationary processes. These are large rotating machines as wind power generators, powerful turbo-generators subjected to inertial and kinematics (seismic) excitations, and etc. Similar structures possess significant vertical stiffness and several times lower stiffness in horizontal direction, because of specific equipment placed under them (columns, support, and etc.) $[10,11]$. As a result of these particularities, it could be observed large deflections and unstable areas of oscillations. Non-stationary vibrations and transitional processes are analyzed. In [12, 13], vibrations of flexible rotor driven by limited power are analyzed. The transition effect as a result of limited power excitation is analyzed by asymptotic methods [14-17] of averaging and the possible areas of unstable vibrations trough resonance transition are estimated as by quasi-static increasing, so by decreasing of the exiting frequency.

The results of the investigations are proved, using numerical procedure for dynamics simulation of rigid and flexible multibody systems [18]. Transient effects are simulated and compared to the results of the analytical investigations, proving excellent agreement. Examples of simulation of the transient effects, vibrations and non-stationary behaviour of the multibody system are presented. The effects of the earthquake excitations of the system behaviour are analyzed. 


\section{ANALYTICAL MOdel of A High SPEEd ROTOR ON A SIZEABLE FLEXIBLE FOUNDATION}

In Fig. 1, the design scheme of a turbo-machine, mounted on a large flexible pillar is depicted. Such design scheme is similar to the steam or wind generator and is very sensible to the external excitations or inaccuracy of the construction as rotor eccentricity, clearances in the bearings, and etc. The pillar (a beam of steel tube) is placed on a basement. On the top of the beam the stator and the rotor of the wind generator are mounted. The rotation is along an axis perpendicular to the plane $X Y$, while the deflections of the system are in the same plane.

In Fig. 1 (b), the kinematics analogue (scheme) is presented and in Fig. 1 (c) the corresponding dynamic model of the system, including the reduced mass and stiffness properties. The system is typical multibody system, compiled of rigid and flexible bodies. Further down, the analytical approach for deriving of the dynamics equations is presented.

The first modal frequency and modal mode of vibration are obtained, taking into account, that the pillar is actually cantilever beam for which the first $\omega_{\text {col }_{1}}$ modal frequencies are computed as follows $[15,19]$ :

$$
\omega_{c o l_{1}}=1.875^{2} \cdot \sqrt{\frac{E . I}{m_{\rho} \cdot H^{4}}},
$$

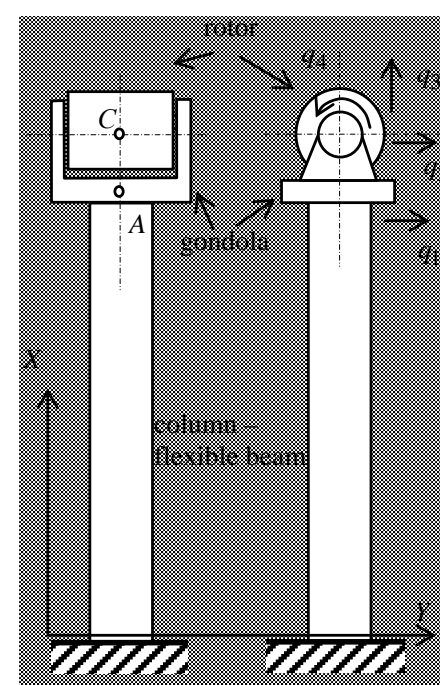

(a) Virtual design scheme

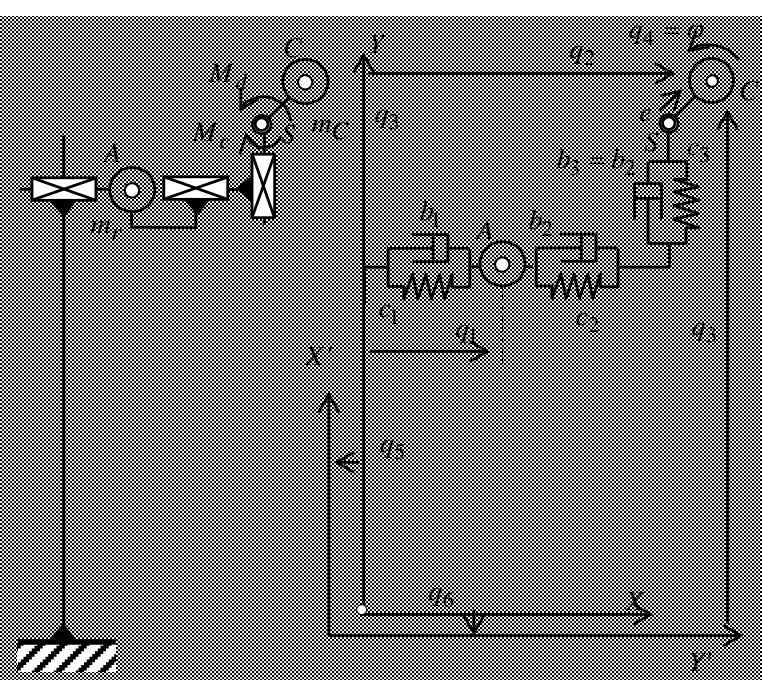

(b) Kiniematic analog (c) Dynamic
model

Fig. 1. Test model of high speed rotating machine mounted on an elastic column. 
where $E$ (all measures are in SI UNITS) is Young modulus of the elasticity; $I$ is the geometrical inertia moment; $m_{\rho}=m_{c o l} / H$ (kilograms per meter, i.e. density along the length) is the distributed mass along the beam length; $H$ is the length of the beam. The mass reduced to the beam tip, $m_{r}$, is to be derived, equalizing the kinetic energy $E_{k}$ of the reduced mass and of all distributed masses by the first natural frequency method of Rely [15], i.e.

(2)

$$
\begin{aligned}
& E_{k}=\frac{1}{2} \cdot m_{r} \cdot u_{11}^{2} \cdot \omega_{c o l_{1}}^{2}=\frac{1}{2} \cdot \omega_{c o l_{1}}^{2} \cdot \sum_{i=1}^{n} m_{i} \cdot u_{1 i}^{2}, \\
& m_{r}=\frac{\sum_{i=1}^{n} m_{i} \cdot u_{i 1}^{2}}{u_{11}^{2}},
\end{aligned}
$$

where $m_{i}=\frac{m_{\rho} . H}{i}=\frac{m_{c o l}}{i}, i=1,2, \ldots, n$ is the mass of the distributed nodes and $u_{i 1}$ is the value of the first modal vector, corresponding to the first natural frequency (Fig. 2). The mass pointed out with $m_{A}$ (point A in Fig. 2) includes the reduced mass of the pillar $m_{r}$ and the mass of the stator (gondola). $m_{C}$ of point $C$ is the mass of the rotor. Taking into account the first modal frequency and the mass reduced to the pillar tip one could estimate $[19,20]$ the stiffness coefficient $c_{1}$ of bending.

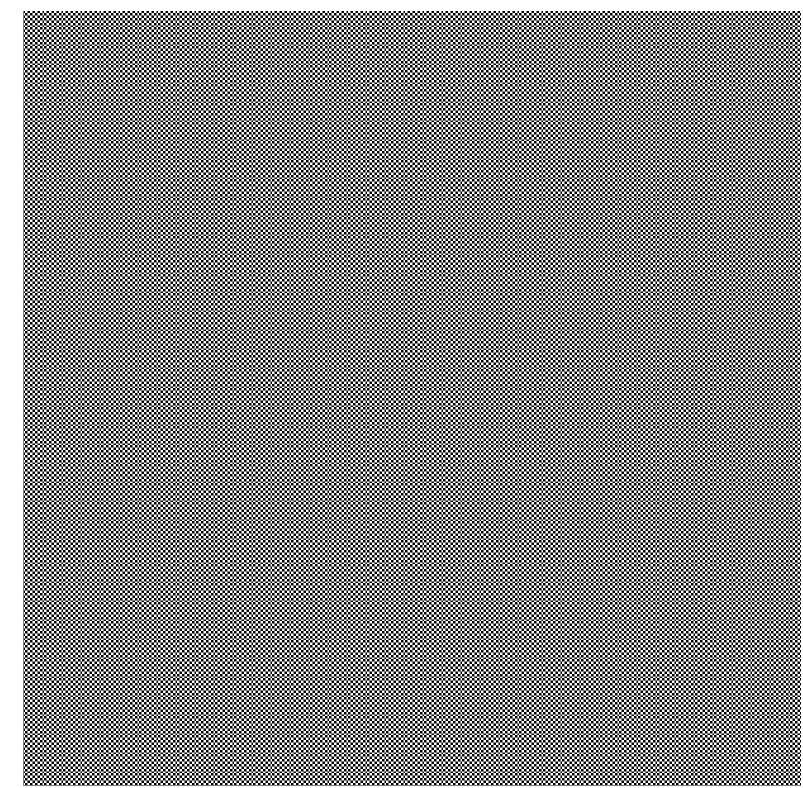

Fig. 2. Algorithm for mass reduction of the column. 


\subsection{DYNAMIC EQUATIONS}

The expressions for the kinetic and potential energy are very simple, since the system is with respect to the absolute coordinates of the rotor mass center, i.e.

$$
\begin{aligned}
T & =\frac{1}{2} m_{A} \cdot \dot{q}_{1}^{2}+\frac{1}{2} J \cdot \dot{q}_{4}^{2}+\frac{1}{2} m_{C} \cdot\left(\dot{q}_{2}^{2}+\dot{q}_{3}^{2}\right), \\
\Pi & =\frac{1}{2} c_{1} \cdot q_{1}^{2}+\frac{1}{2} c_{2}\left(g_{2}-q_{1}-e \cdot \cos q_{4}\right)^{2}+\frac{1}{2} c_{2}\left(q_{2}-e \cdot \sin q_{4}\right)^{2}, \\
c_{2} & =c_{3}, \\
\Phi & =\frac{1}{2} b_{1} \cdot \dot{q}_{1}^{2}+\frac{1}{2} b_{2} \cdot \dot{q}_{2}^{2}+\frac{1}{2} \cdot b_{3} \dot{q}_{3}^{2} .
\end{aligned}
$$

As it could be seen from the kinetic energy, no quadratic terms for the coordinate velocities would appear after differentiation, according to the Lagrange dynamics equations.

Using the Lagrange dynamics equation and the expressions for the kinetic and potential energies, Eq. (3), one obtains the following dynamic equations for the system in Fig. 1 (c):

$$
\begin{aligned}
& m_{A} \cdot \ddot{q}_{1}+c_{0} \cdot q_{1}+b_{0} \cdot \dot{q}_{1}-c_{2} \cdot q_{2}=-c_{2} \cdot e \cdot \cos \left(q_{4}\right)+c_{0} \cdot q_{5}+b_{0} \cdot \dot{q}_{5} ; \\
& m_{C} \cdot \ddot{q}_{2}-c_{2} \cdot q_{1}+c_{2} \cdot q_{2}+b_{2} \cdot \dot{q}_{2}=c_{2} \cdot e \cdot \cos \left(q_{4}\right)+c_{2} \cdot q_{5}+b_{2} \cdot \dot{q}_{5} ; \\
& m_{C} \cdot \ddot{q}_{3}+c_{2} \cdot q_{3}+b_{2} \cdot \dot{q}_{3}=c_{2} e \cdot \sin \left(q_{4}\right)+c_{2} \cdot q_{6}+b_{2} \cdot \dot{q}_{6} ; \\
& J_{C} \cdot \ddot{q}_{4}=M_{d}\left(\dot{q}_{4}\right)-M_{c}\left(\dot{q}_{4}\right)-c_{2} \cdot e \cdot\left(q_{2}-q_{1}\right) \sin \left(q_{4}\right)+c_{2} \cdot e \cdot q_{3} \cdot \cos \left(q_{4}\right),
\end{aligned}
$$

where $c_{2}=c_{3}, b_{2}=b_{3}, b_{0}=b_{1}+b_{2} ; c_{0}=c_{1}+c_{2} ; q_{5}$ and $q_{6}$ are kinematics (seismic) excitations, along axes $X$ and $Y$ to a new position $X^{\prime}$ and $Y^{\prime}$, respectively; $M_{d}$ - driving torque; $M_{c}$ - resistant torque; $c_{1}, c_{2}, c_{0}=c_{1}+c_{2}$ are the stiffness coefficients; $b_{1}, b_{2}, b_{0}=b_{1}+b_{2}$ are damping coefficients; $q_{i}, i=(1,2,3,4,5,6)$ are generalized coordinates; $J_{C}$ is mass inertia moment of the rotor; $e$ is the rotor eccentricity. The damping coefficient of the column $b_{1}$ is computed using that one for the first modal frequency of the column and amplitude decrement of the free vibrations [21]. As it is pointed out in [22], the gravity could cause influence on the magnitude of the modal frequencies. In the dynamic equations, the gravity is not taken into consideration, since the geometrical cross section inertia is very high (at about $0.061\left[\mathrm{~m}^{4}\right]$ ) and the influence of the gravity on the frequency and the amplitude is very low - less that $1 \%$ [15]. Further down, the analytical solution of Eqs. (4) does not discuss external excitations, while they are considered in the next section that describes the numerical procedure. Equations (4) are derived with respect to the absolute coordinates $X, Y$, as shown in Fig. 1(c), where the position coordinates 
of the rotor, $C$, is represented as $q_{2}, q_{3}$ and the rotation is represented as $q_{4}$. As a consequence, the system implements plane motion. Hence, the quadratic terms for the generalized velocities do not appear in the dynamic equations, Egs. (4).

\subsection{Modal FREQUenCIES AND MOdAL Vectors}

With $q_{5}(t)=0, \dot{q}_{5}(t)=0, q_{6}(t)=0, \dot{q}_{6}(t)=0$ (external excitations are not taken into account and are considered in Section 3), the frequency spectrum of the conservative system of differential equations, that corresponds to the system, Eqs. (4), is obtained, using the relations:

$$
\left.\begin{array}{c}
\omega_{1}^{2} \\
\omega_{2}^{2}
\end{array}\right\} \rightarrow\left|\begin{array}{cc}
c_{0}-m_{A} \cdot \omega^{2} & -c_{2} \\
-c_{2} & c_{2}-m_{C} \cdot \omega^{2}
\end{array}\right|=0 ; \quad \omega_{3}^{2}=\frac{c_{2}}{m_{C}} .
$$

It should be pointed out that $\omega_{3}$, from physical point of view, is the critical angular velocity of the shaft for motionless mass $m_{A}$. The modal vectors of the vibrations, corresponding to $\omega_{i}$, are as follows:

$$
\{u\}_{i}=\left\{\begin{array}{l}
u_{1 i} \\
u_{2 i}
\end{array}\right\}=u_{1 i} \cdot\left\{\begin{array}{c}
1 \\
\frac{u_{2 i}}{u_{1 i}}
\end{array}\right\}=u_{1 i} \cdot\left\{\begin{array}{c}
1 \\
\frac{-c_{2}}{c_{2}-m_{C} \cdot \omega_{i}^{2}}
\end{array}\right\}, \quad(i=1,2) .
$$

\subsection{QUASI-NORMAL COORDINATES}

The quasi-normal coordinates of Eqs. (4), in the absence of kinematics disturbances, are presented by the following equation system:

$$
\begin{aligned}
& \ddot{x}_{1}+\omega_{1}^{2} \cdot x_{1}=-f_{1} \cdot \cos \left(q_{4}\right)-2 \cdot n_{1} \cdot \dot{x}_{1} ; \\
& \ddot{x}_{2}+\omega_{2}^{2} \cdot x_{2}=+f_{2} \cdot \cos \left(q_{4}\right)-2 \cdot n_{2} \cdot \dot{x}_{2} ; \\
& \ddot{x}_{3}+\omega_{3}^{2} \cdot x_{3}=-f_{3} \cdot \sin \left(q_{4}\right)-2 \cdot n_{3} \cdot \dot{x}_{3} ; \\
& \ddot{q}_{4}=\frac{1}{J_{0}}\left\{M_{d}\left(\dot{q}_{4}\right)-M_{c}\left(\dot{q}_{4}\right)+f_{4} \cdot\left(x_{2}-x_{1}\right) \cdot \sin \left(q_{4}\right)+f_{4} \cdot x_{3} \cdot \cos \left(q_{4}\right)\right\},
\end{aligned}
$$

where the physical coordinates from Eqs. (4) are

$$
\begin{aligned}
& q_{i}(t)=\frac{1}{\Im_{i}}\left[m_{A} \cdot u_{i k} \cdot x_{1}(t)+m_{C} \cdot u_{2 k} \cdot x_{2}(t)\right], \\
& f_{i}=\frac{c_{2} \cdot e \cdot \sum_{1}^{2} u_{i k}}{\Im_{i}}, \quad i=1,2, \quad k=1,2 ; \\
& \Im_{i}^{0}=m_{A} \cdot u_{1 i}^{2}+m_{C} \cdot u_{2 i}^{2}, \quad i=1,2, \quad k=1,2 ;
\end{aligned}
$$




$$
\begin{aligned}
& f_{3} \equiv f_{4}=c_{2} \cdot e, \quad 2 . n_{1}=\frac{b_{0} \cdot u_{11}^{2}+b_{2} \cdot u_{21}^{2}}{\Im_{1}^{0}} ; \\
& 2 . n_{2}=\frac{b_{0} \cdot u_{12}^{2}+b_{2} \cdot u_{22}^{2}}{\Im_{2}^{0}} ; \quad 2 . n_{3}=\frac{b_{2}}{m_{C}}, \quad q_{3}=x_{3} .
\end{aligned}
$$

In Eqs. (7), the following relations in the vicinity of the resonances hold:

$$
\begin{aligned}
& x_{i}=A_{i} \cdot \cos \left(q_{4}+\varsigma_{i}\right) ; \\
& \frac{d x_{i}}{d t}=-A_{i} \cdot \omega_{i} \cdot \sin \left(q_{4}+\varsigma_{i}\right) ; \\
& \frac{d q_{4}}{d t}=\theta, \quad i=1,2,3 .
\end{aligned}
$$

where $A_{i}, \varsigma_{i}$ and $\theta$ are slowly changing functions of time, according to the asymptotic method of Bogoljubov and Mitropolski (function with small change of the first derivative in a narrow frequency range).

\subsection{Method OF AVERAGING}

Following the asymptotic method of Bogoljubov [14], further published in the book of Bogoljubov and Mitropolski [23], for slowly changing of amplitudes, driving frequencies and phases of vibrations and using the relations, Eqs. (8), one obtains, for example, for the first resonance $\omega_{1}$, that

$$
\begin{aligned}
& x_{1}=A_{1} \cdot \cos \left(q_{4}+\varsigma_{1}\right) ; \\
& \frac{d x_{1}}{d t}=-A_{1} \cdot \omega_{1} \cdot \sin \left(q_{4}+\varsigma_{1}\right) ; \\
& \frac{d q_{4}}{d t}=\theta,
\end{aligned}
$$

where, as it was pointed out, above $A_{1}, \varsigma_{1}$ and $\theta$ are slowly changing functions of time. With these conditions, $\dot{x}_{1}$ from the first equation of Eqs. (9), is equal to

$$
\frac{d x_{1}}{d t}=\frac{d A_{1}}{d t} \cdot \cos \left(q_{4}+\varsigma_{1}\right)-A_{1} \cdot\left(\theta+\frac{d \varsigma_{1}}{d t}\right) \cdot \sin \left(q_{4}+\varsigma_{1}\right) .
$$

Differentiating the second equation of Eqs. (9). with respect to time, one obtains

$$
\frac{d^{2} x_{1}}{d t}=-\frac{d A_{1}}{d t} \cdot \omega_{1} \sin \left(q_{4}+\varsigma_{1}\right)-A_{1} \cdot\left(\theta+\frac{d \varsigma_{1}}{d t}\right) \cdot \omega_{1} \cdot \cos \left(q_{4}+\varsigma_{1}\right)
$$


After substitution of $x_{1}, \dot{x}_{1}$ from Eqs. (8) and $\ddot{x}_{1}$ from Eq. (11) in the first equation of Eqs. (6), one obtains the following dependence:

$$
\begin{aligned}
& -\frac{d A_{1}}{d t} \cdot \omega_{1} \cdot \sin \left(q_{4}+\varsigma_{1}\right)-\frac{d \varsigma_{1}}{d t} \cdot A_{1} \cdot \omega_{1} \cdot \cos \left(q_{4}+\varsigma_{1}\right)= \\
= & \varepsilon\left[\left(\theta-\omega_{1}\right) \cdot \omega_{1} \cdot A_{1} \cdot \cos \left(q_{4}+\varsigma_{1}\right)-f_{1} \cdot \cos \left(q_{4}\right)-2 \cdot n_{1} \cdot A_{1} \cdot \omega_{1} \sin \left(q_{4}+\varsigma_{1}\right)\right]
\end{aligned}
$$

Solving simultaneously Eq. (9) (the second one of Eqs. 8) and Eq. (12) as a system of two equations with two unknowns for $\frac{d A_{1}}{d t}$ and $\frac{d \varsigma_{1}}{d t}$, rewriting Eq. (12) and changing the variables $d t$ by $d q_{4}\left(q_{4}\right.$, is $\theta . t+$ constant), one obtains the following equation system:

$$
\begin{aligned}
& \frac{d A_{1}}{d q_{4}}= \frac{\varepsilon}{\omega_{1} \cdot \theta}\left[f_{1} \cdot \cos \left(q_{4}\right) \cdot \sin \left(q_{4}+\varsigma_{1}\right)-2 \cdot n_{1} \cdot A_{1} \cdot \omega_{1} \cdot \sin ^{2}\left(q_{4}+\varsigma_{1}\right)\right] ; \\
& \frac{d \varsigma_{1}}{d q_{4}}=\frac{\varepsilon}{\theta}\left[\left(\theta-\omega_{1}\right)-f_{1} \cdot \frac{1}{A_{1} \cdot \omega_{1}} \cdot \cos \left(q_{4}\right) \cdot \cos \left(q_{4}+\varsigma_{1}\right)-\right. \\
&\left.\quad-2 \cdot n_{1} \cdot \sin \left(q_{4}+\varsigma_{1}\right) \cdot \cos \left(q_{4}+\varsigma_{1}\right)\right] ; \\
& \frac{d \theta}{d q_{4}}=\frac{\varepsilon}{J \cdot \theta}\left[M_{d}(\theta)-M_{c}(\theta)+f_{4} \cdot A_{1} \cdot \cos \left(q_{4}+\varsigma_{1}\right) \cdot \sin \left(q_{4}\right)\right],
\end{aligned}
$$

where $\theta$ appears in the denominator. Assuming that

$$
q_{4}=\Omega . t+\varepsilon \cdot \Phi(t)
$$

where $\Omega$ is the rotor angular velocity, $\Phi(t)$ is slowly changing function of time $t$, one can change the variables in the equation system, Eqs. (7). By this means and for small disturbances in the vicinity of the resonances $\omega_{i},(i=1,2,3)$, the approximate solutions are in the following form:

$$
\begin{aligned}
& \theta=\Omega+\varepsilon \cdot \mathrm{E}_{1} ; \\
& A_{i}=a_{i}+\varepsilon \cdot \mathrm{E}_{2} ; \\
& \varsigma_{i}=\xi_{i}+\varepsilon \cdot \mathrm{E}_{3}, \quad i=1,2,3,
\end{aligned}
$$

where $\varepsilon \cdot \mathrm{E}_{i}, A_{i}, \varsigma_{i}, i=1,2,3$, and $\theta$ are slowly changing functions of time. Averaging the values of Eqs. (13) for one period of time, the following equation system is 
derived:

$$
\begin{aligned}
& \frac{d A_{1}}{d q_{4}}=\frac{1}{2 . \pi} \int_{0}^{2 . \pi} \frac{d A_{1}}{d q_{4}} d q_{4}=f_{1} \cdot \sin \left(\xi_{1}\right)+2 \cdot n_{1} \cdot a_{1} \cdot \omega_{1} ; \\
& \frac{d \varsigma_{1}}{d q_{4}}=\frac{1}{1 \cdot \pi} \int_{0}^{2 \cdot \pi} \frac{d \varsigma_{1}}{d q_{4}} \cdot d q_{4}=\left(\Omega-\omega_{1}\right)+\frac{1}{2} \cdot \frac{f_{1}}{a_{1} \cdot \omega_{1}} \cdot \cos \left(\xi_{1}\right) ; \\
& \frac{d \Omega}{d q_{4}}=\frac{1}{2 \cdot \pi} \int_{0}^{2 . \pi} \frac{d \theta}{d q_{4}} \cdot d q_{4}=M_{\partial}(\Omega)-M_{c}(\Omega)-\frac{f_{4}}{2} \cdot \cos \left(\xi_{1}\right) .
\end{aligned}
$$

In quasi-stationary mode of motion, it is properly the left sides of the Eqs. (16) to be set to zero with which the amplitudes $a_{1}$, the phase $\xi_{1}$, and the additional resistance torques in the zone of the first resonance to be estimated, i.e.

$$
\begin{gathered}
f_{1} \cdot \sin \left(\xi_{1}\right)+2 \cdot n_{1} \cdot a_{1} \cdot \omega_{1}=0 ; \\
\left(\Omega-\omega_{1}\right)+\frac{1}{2} \cdot \frac{f_{1}}{a_{1} \cdot \omega_{1}} \cdot \cos \left(\xi_{1}\right)=0 ; \\
M_{d}(\Omega)=M_{c}(\Omega)-\frac{f_{4}}{2} \cdot a_{1} \cdot \sin \left(\xi_{1}\right)=M_{c}(\Omega)+\Delta L_{1}(\Omega) .
\end{gathered}
$$

Using the same procedure for the second $\omega_{2}$ and third $\omega_{3}$ resonances, similar results of these of Eqs. (17) are obtained, i.e.

$$
\begin{gathered}
f_{i} \cdot \cos \left(\xi_{i}\right)+2 \cdot n_{i} \cdot a_{i} \cdot \omega_{i}=0 \\
\left(\Omega-\omega_{i}\right)+\frac{1}{2} \cdot \frac{f_{i}}{a_{i} \cdot \omega_{i}} \cdot \sin \left(\xi_{i}\right)=0 ; \\
M_{d}(\Omega)=M_{c}(\Omega)-\frac{f_{4}}{2} \cdot a_{i} \cdot \sin \left(\xi_{i}\right)=M_{c}(\Omega)+\Delta L_{i}(\Omega), \quad i=2,3 .
\end{gathered}
$$

The amplitudes of the vibrations in the vicinity of each of the resonances, $\omega_{i} \equiv \Omega$ is expressed by the following dependences:

$$
a_{i}(z)=\frac{f_{i}}{2 \cdot \omega_{i} \cdot \sqrt{(1-z)^{2}+\nu_{i}^{2}}} ; \quad z=\frac{\Omega}{\omega_{i}} ; \quad \nu_{i}=\frac{n_{i}}{\omega_{i}}, \quad i=1,2,3 .
$$

The additional resistance torque $\Delta L_{i}\left(z_{i}, \omega_{i}\right)$, in case of small disturbances $\left(\omega_{i}-\Omega\right)$, respectively $(1-z)$, is expressed as follows:

$$
\Delta L_{i}\left(z_{i} \cdot \omega_{i}\right)=\frac{f_{i} \cdot f_{4} \cdot \nu_{i}}{4 \cdot \omega_{i}^{2}\left[(1-z)^{2}+\nu_{i}^{2}\right]}, \quad i=1,2,3
$$


and the common resistant torque $\Delta L_{i}\left(z, \omega_{i}\right)$ is

$$
L\left(z, \omega_{i}\right)=M_{c}\left(z, \omega_{i}\right)+\Delta L_{i}\left(z, \omega_{i}\right)=k \cdot \omega_{i}^{2} \cdot z_{i}^{2}+\frac{f_{i} \cdot f_{4}}{4 \cdot \omega_{i}^{2}} \cdot \frac{\nu}{\left[\left(1-z^{2}\right)+\nu_{i}^{2}\right]} .
$$

\subsection{EXAMPLE}

The frequency spectrum of the pillar and the common resistant torque as well as, the amplitudes, modal vectors, stability of the vibrations and others in quasi-stationary transitional modes are calculated, based on the theoretical results and conclusions and for the geometrical and physical parameters of a turbo-generator with power about $350 \times 10^{3}$. The following input data are used: the pillar $-\mathrm{a}$ beam of steel tube with height $H=20$; density $\rho=8.1 \times 10^{3}$; outer and inner diameters $D_{0}=2, D_{1}=1.96$, respectively; mass of the gondola (stator) $m^{*}=2.3 \times 10^{3}$; distributed beam mass $m_{i}=1 \times 10^{3}$; beam rigid body mass reduced to the beam tip $-m_{r}=9.7 \times 10^{3}$, $m_{A}=m_{r}+m^{*}$ in accordance with Figs. 1, 2 and Eq. 2; stiff and damping coefficients of the beam $c_{1}=9.6 \times 10^{6}, b_{1}=(10 \div 50) \times 10^{3}$, respectively; rotor mass (mass in point $C$ ) $m_{C}=1.6 \times 10^{3} ; c_{2}=0.4 \times 10^{6}$; mass inertia moment of the rotor

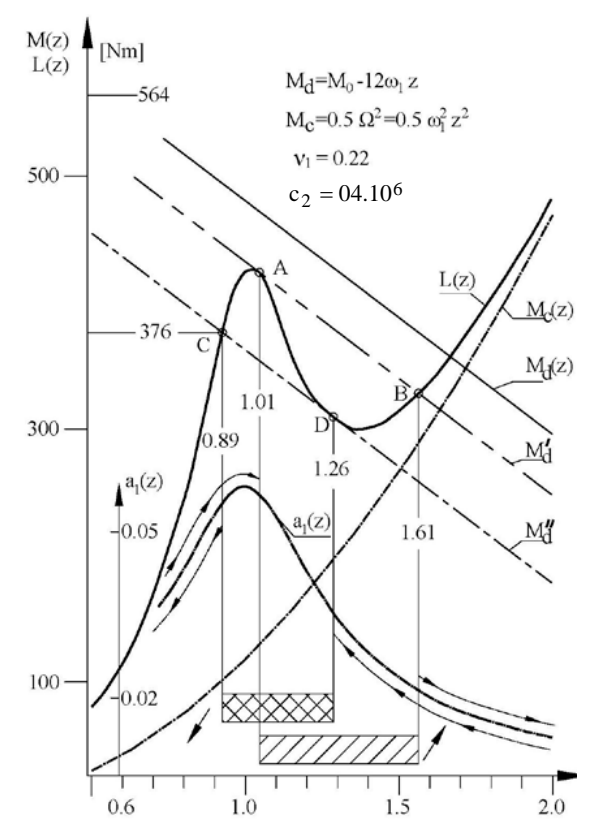

Fig. 3. Results for $a_{1}(z), M_{d}(z), L(z)$ in case of transition through the first resonance for $\nu_{1}=0.22$.

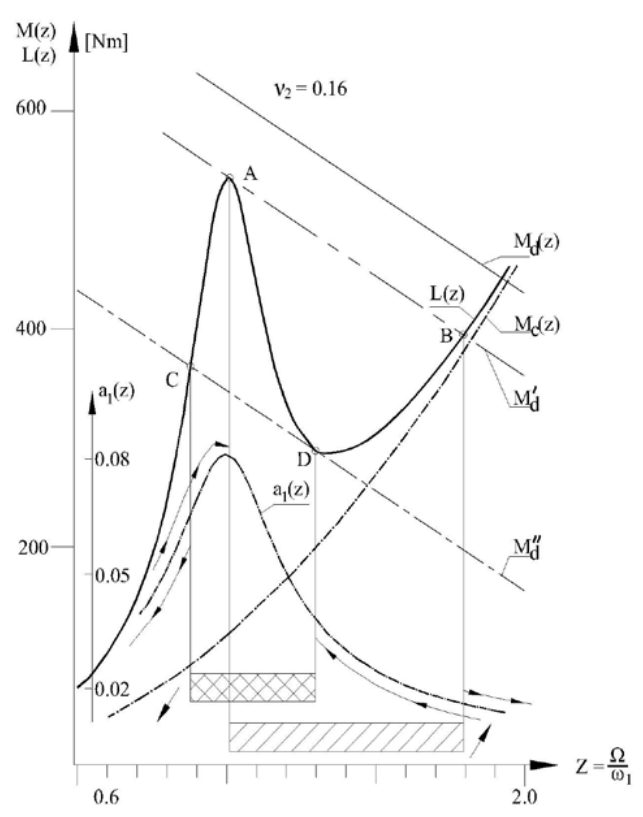

Fig. 4. Results for $a_{1}(z), M_{d}(z), L(z)$ in case of transition through the first resonance for $\nu_{1}=0.16$. 
$J_{C}=100$; rotor eccentricity $e=0.002 ; \omega_{3}=15.8 ; \omega_{c o l}=31.45$ (Eq. 1). The driving $M_{d}$ and resistant $M_{c}$ torques are of the type

$$
\begin{aligned}
& M_{c}\left(\dot{q}_{4}\right) \approx M_{c}(\Omega)=0.5 \cdot \Omega^{2}=0.5 \cdot \omega_{1}^{2} \cdot z^{2} ; \\
& M_{d}=M_{0}-12 . \omega_{1} \cdot z ; \\
& M_{0}=300 . \tau, \quad(1.7 \leq \tau \leq 17),
\end{aligned}
$$

where $M_{0}$ is the torque for zero angular velocity and $\tau$ is coefficient for parallel translation of the driving torque $\left(M_{d}^{\prime}, M_{d}^{\prime \prime}, M_{d}^{\prime \prime \prime}\right.$ in Figs. 3 and 4.

In Figs. 4 and 5, the results for $a_{1}(z), M_{d}(z), L(z)$ are depicted in case for transition through the first resonance $\Omega=\omega_{1}$, i.e. $z=1, \omega_{1}=26$ for two different values of $\nu_{1}$, i.e. $\nu_{1}=0.22 ; \nu_{1}=0.16$, where $\nu_{1}=\frac{n_{1}}{\omega_{1}}$.

Table 1. Computational results for the case of stiff shaft $c_{2}=0.4 \times 10^{6}$

\begin{tabular}{|c|c|c|c|c|c|}
\hline \multirow{2}{*}{ No. of the resonance } & $f_{i}$ & $2 . n_{i}$ & $\nu_{1}$ & $\Im_{i}^{0} \times 10^{3}$ & $\omega_{i}$ \\
\cline { 2 - 6 } & {$\left[\mathrm{m} / \mathrm{s}^{2}\right]$} & {$\left[\mathrm{s}^{-1}\right]$} & {$[-]$} & {$[\mathrm{kg}]$} & {$\left[\mathrm{s}^{-1}\right]$} \\
\hline 1 & 0.065 & 6.25 & 0.22 & 14.1 & 26 \\
\hline 2 & 0.356 & 13.75 & 0.16 & 2.7 & 59.1 \\
\hline
\end{tabular}

Varying the driving torque for zero velocity, $M_{d_{0}}$, i.e. changing $\tau$, the crosspoints between the driving torque and the generalized resistant torque in the resonance domain are changing. In other words, parallel translation of the driving torques is implemented and for quasi-stationary increasing, decreasing of the rotor velocity, respectively, it could be observed violation of the obvious condition:

$$
\frac{d\left[M_{d}(z)-L(z)\right]}{d z}<0,
$$

for vibration stability. As a result of that, it could be observed in the frequency range, from point $A$ to point $B$ for increasing of $\Omega$, respectively $z$, as well as, from point $D$ to point $C$ (Figs. 3 and 4 for different values of the dissipation) in case of decreasing of $\Omega$, collapse of the vibrations, i.e., instability.

The results are obtained for two cases. The first one discusses extremely elastic shaft with stiff coefficient $c_{2}=0.4 \times 10^{6}$ and damping $b_{2}=11 \times 10^{3}$ (Fig. 3). The second one is several times stiffer beam.

For the second case (stiff shaft with $c_{2}=5 . \times 10^{6}$ and $b_{2}=25 . \times 10^{3}$ ) similar collapses of the vibrations during the transitions via the resonances $\omega_{1}$ and $\omega_{2}$ are not observed, because of the small ratios, i.e.

$$
\frac{\Delta L_{1_{\max }}}{M_{c}\left(\omega_{1}\right)}=\frac{f_{4}}{2} \cdot \frac{a_{1_{\max }}}{M_{c}\left(\omega_{1}\right)}=0.11 ; \quad \frac{\Delta L_{2_{\max }}}{M_{c}\left(\omega_{2}\right)}=\frac{f_{4}}{2} \cdot \frac{a_{2_{\max }}}{M_{c}\left(\omega_{2}\right)}=0.07,
$$


where $a_{1_{\max }}=0.010, a_{2_{\max }}=0.027 . M_{c}\left(\omega_{1}\right)=454, M_{c}\left(\omega_{2}\right)=1930$. The natural frequencies $\omega_{1}, \omega_{2}$ from Table 1 are used for control benchmarks, as well as, for comparison with the results obtained by numeric simulation.

\section{VERIFICATION OF THE ANALYTICAL RESULTS USING NUMERICAL METHOD FOR DYNAMICS SIMULATION}

The numerical approach to dynamics simulation of rigid and flexible multibody systems enables many nonlinear effects as contact, friction, external excitations (seismic disturbances) to be taken into account. The numerical methods are mainly based on the quasi-static dynamics approach, for which the inertia forces are assumed as external loading. The dynamic equations of flexible elements are derived, using finite element discretization. In [18], novel generalized Newton-Euler dynamic equations are proposed, in which the dependences for the inertia terms are valid as for rigid bodies, so for flexible elements. The structure of Fig. 1 is analyzed using the program system, based of the numerical approach created on the basis of the dynamic equations proposed. The elastic column is discretized on three beam elements, taking into account bending and rotation of the beam cross section. So, the dynamic model is precise and the analytical solution (Section 2) will be analysed. The difference between the two approaches is that for the analytical solution no rotation of the beam cross section is assumed as a coordinate.

The numerical results presented in Fig. 5 (a) fully comply with the computations in the previous section. The first and the second natural frequencies are almost equal, obtained analytically $\left(\omega_{1}=26, \omega_{2}=59.1\right.$, see Table 1$)$, and numerically $\left(\omega_{1}=\right.$ $24.04, \omega_{2}=61.58$, see Fig. 5 a). These results prove that the approach to the pillar mass reduction is appropriate and, as a result, the neglecting of the beam cross section rotation does not cause significant influence on the results.

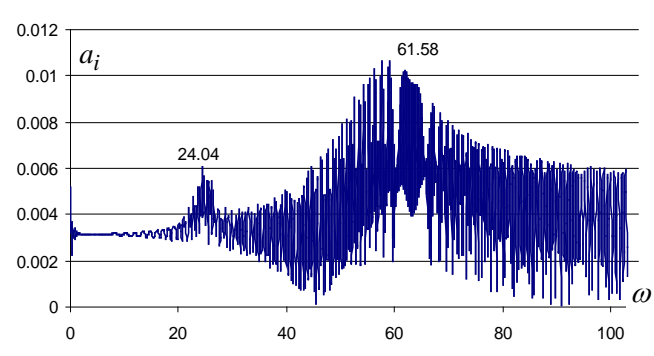

(a) no seismic excitations

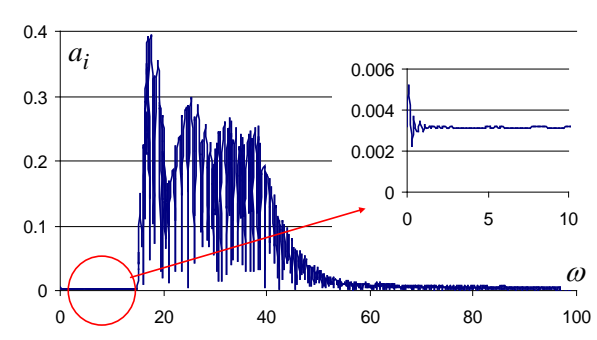

(b) in case of seismic excitations

Fig. 5. Rotor displacements: (a) operational time $t=80 \mathrm{sec}$. $\left(0 \leq \omega_{e} \leq 120\right)$; (b) subject to seismic excitation within the time interval $5 \leq t \leq 12$. 
The same numerical procedure is applied for dynamic simulation of the structure of Fig. 1 for which seismic excitation is imposed with ground motion $q_{5}=$ $-\frac{\mathrm{g}}{\pi}+\frac{\mathrm{g}}{\pi} \cdot \cos (\pi \cdot t), q_{6}=0$, where $\mathrm{g}$ is the earth acceleration. The time of the earthquake is 7 seconds. The results for the rotor displacements are depicted in Fig. 5 b. The combination of the analytical and the numerical methods is an approach to solution of two major tasks. Deriving explicit functions that cause the influence of the design parameters to the system behaviour is a powerful tool for the design process. Numerical methods, as in this case, could check the system imposed in different phenomena and nonlinear effects. The future aim of the investigations is to derive analytical solution in case of external excitations and consequential comparison to the numerical computations.

\section{Conclusions}

It is necessary, independently form the gradient direction of the angular velocity perturbations, the stiffness, $c_{2}$, of the rotor shaft to be increased, in order to avoid unstable frequencies ranges in the rotor systems during transmissions via resonance conditions.

The pillar stiffness $c_{1}$ and of the rotor shaft $c_{2}$ are to be of the same order. The coincidence of the computational results obtained by the numerical procedure for the modal vectors and frequencies of the pillar and the whole system, as well as, of the vibration amplitudes for quasi-stationary transitions for the resonance period is verification for precision and effectiveness of the geometrical approach proposed.

\section{ACNOWLEDGEMENT}

The authors acknowledge the financial support of Ministry of Education, Youth and Science, contract No. DUNC 01/3 - 2009.

\section{REFERENCES}

[1] BAChelet, L., N. DRIOT, G. FerRaris. Rotors under Seismic Excitation: A Spectral Approach, In: Proc. of the 7th IFToMM International Conference on Rotor Dynamics, Vienna, Austria, Vienna 25-28 September, 2006.

[2] LeE, A. S., B. O. KIM, Y-C. Kim. A Finite Element Transient Response Analysis Method of a Rotor-bearing System to Base Shock Excitations using the State-space Newmark Scheme and Comparisons with Experiments. Journal of Sound and Vibration, 297 (2006), No. 3-5, 595-615.

[3] Kang, Y., Y-P. Chang, J-W Tsai, L-H. Mu, Y-F. Chang. An Investigation in Stiffness Effects on Dynamics of Rotorbearing-foundation Systems. Journal of Sound and Vibration, 231 (2000), No. 2, 343-374. 
[4] Duchemin, M. A., G. Berlioz, N. Ferraris. Dynamic Behaviour and Stability of a Rotor under Base Excitation. Transactions of the ASME, Journal Of Vibration and Acoustics, 128 (2006), 576-585.

[5] Leontiev, M. K., S. A. Degtiarev. Dynamics of Rotor Systems under Seismic Excitation, Vestnik Voronejkogo Gosudarstvenogo Tehnicheskogo Universitetaa (English Version - http://www.alfatran.com/publications.shtml), 8 (2012), No. 71, 1 - 6.

[6] Zaki, D. M., B. Sebastien, D. Regis. Steady-state Dynamic Behaviour of an Onboard Rotor under Combined Base Motions. Journal of Vibration and Control, SAGE Publications, 20 (2014), No. 15, 2254-2287.

[7] Mukherjee, A., V. Rastogi, A. DasGupta. A Study of a Bi-Symmetric Electromechanical System through Umbra Lagrangian generated by Bond Graphs, and Noether's Theorem. Simulation, 83 (2007), No. 9, 611-630.

[8] Mukherjee, A., V. Rastogi, A. DasGupta. Extension of Lagrangian-Hamiltonian Mechanics for Continuous Systems-Investigation of Dynamics of a One Dimensional Internally Damped Rotor driven through a Dissipative Coupling. Nonlinear Dynamics, 58 (2009), No. 1, 107-127.

[9] Noether, E. Invariant Variation Problems (translated by Mort Tavel) Transport Theory and Statistical Physics, 1 (1971), No. 3, 186-207.

[10] Negm, H. M., K. Y. MaAlowi. Structural Design Optimization of Wind Turbine Towers, Computers and Structures, Elsevier, 2000.

[11] Burton, T., D. Sharpe. Wind Energy Handbook, John Wiley \& Sons. Ltd., 2001.

[12] Koterat, T. Vibration of Flexible Rotor Driven by Limited Torque through its Critical Speed. Bull ASME, 17 (1974), No. 108, 686 - 692.

[13] TAkuzo, I., K. Hiroshi, K. Ryoji. Vibrations through Critical Speeds of Asymmetric Rotor with Limited Power. Trans. Japan Society of Mechanical Engineering, 40 (1974), No. 335, 1908-1913.

[14] Bogoljubov, N. N. Theory of Disturbances in Nonlinear Mechanics, Collection of Papers of the Institute of Structural Mechanics (in Russian), 14 (1950), 9-34.

[15] Biderman, V. L. Theory of Mechanical Vibrations (in Russian), Moscow, RHD (Reguljarnaja i Haoticheskaja Dinamika), 2009.

[16] Jivkov, V., E. Zahariev. Stability and Non-Stationary Vibrations of Rotor in Elasto - Viscous Field. Mechanics Based Design of Structures and Machines, Taylor \& Francis Group, 42 (2014), No. 1, 35-55.

[17] Jivkov, V. Stability of Steady-State Vibrations of a Rotor in Homogeneous ElastoViscousmField. J. Theor. Appl. Mec., 40 (2010), No. 1, 3-12.

[18] ZAHARIEV, E. Generalized Newton - Euler Equations in Rigid and Flexible Multibody System Dynamics. Journal of Theoretical and Applied Mechanics, Bulgarian Academy of Sciences, 34 (2004), No. 1, 21-26.

[19] BABAKov, I. Theory of Vibrations (in Russian), Moskva, Nauka, 1968.

[20] Meirovich, L. Elements of Vibration Analysis, McGraw-Hill, 1986. 
[21] Hyo-Joon, Kim, Yoo Wan-Suk, OK Jin-Kyu, Kang Dong-Woo. Parameter Identification of Damping Models in Multibody Dynamic Simulation of Mechanical Systems. Multibody System Dynamics, 22 (2009), 383-398.

[22] RaO, S. S. Mechanical Vibrations, 3rd Ed., Addison-Wesley, 1995.

[23] Bogolliubov, N., Y. Mitropolski. Asymptotic Methods in the Theory of Nonlinear Oscillations, New York, Cordon and Breach Science Publication, 1961. 